\title{
Using CRISPR/Cas9 technology to create genetically modified rabbits
}

\author{
V.A. Ezerskiy*, T.P. Trubitsyna, O.B. Zhukova, E.M. Koloskova, N.V. \\ Belova
}

\begin{abstract}
All-Russia Research Institute of Animal Physiology, Biochemistry, and Nutrition, Branch of Ernst Russian Institute of Animal Breeding Federal Science Center for Animal Husbandry, Federal State Badgetary Scientific Institution (248010 Borovsk, p. Institute, Russian Federation)

*corresponding author: ez.vadim@yandex.ru,
\end{abstract}

Journal of Livestock Science (ISSN online 2277-6214) 12: 1-7

Received on 14/8/2020; Accepted on 13/11/20; Published on 3/1/2021

doi. 10.33259/JLivestSci.2021.1-7

\begin{abstract}
The creation of genetically modified animals is becoming an increasingly popular area of scientific and technological progress, primarily for the needs of health and food problems. With the advent of modern genetic engineering methods based on endonucleases, it became possible to obtain animals with high-precision genomic modifications and well-predicted new properties. The creation of self-replicating transgenic animals-bioreactors that secrete biologically active proteins in milk is a promising direction of modern biotechnology. The rabbit is the smallest animal used both in laboratory practice and in animal breeding, from which recombinant pharmaceutical proteins can be obtained on an experimental and industrial scale. Using CRISPR/Cas9 technology, many rabbits have been created in the world - biomodels of human diseases (with gene knockout, transgenic) and producers of human proteins. Rabbits can be effectively used as bioreactors. Whey acidic protein (WAP) is the main protein of whey, its content in rabbit milk is about $15 \mathrm{~g} / \mathrm{l}$, which makes the WAP gene promising for replacing the gene of biologically active protein using the CRISPR/Cas9 system.

A strategy was developed for site-specific modification of the rabbit WAP gene using CRISPR/Cas9 technology. A plasmid containing the 5' and 3' arms of the WAP rabbit gene homology was created. At the junction of the arms homology, a site for the restriction enzyme EagI was introduced. The reporter protein gene was embedded on the EagI site. A strategy for making double-stranded cuts in the WAP gene was developed and its derivatives encoding Cas9 endonuclease and guiding RNA were obtained. The aim of the study was to evaluate the effectiveness of using the green fluorescent protein (EGFP) gene under the cytomegalovirus promoter in a DNA matrix containing homology arms to the WAP gene as an indicator of homologous directed recombination repair (HDR).
\end{abstract}

Key words: rabbits, biomodel, CRISPR/Cas9, microinjection, whey acid protein, in vitro, embryo. 


\section{Introduction}

The main goals of obtaining genetically modified (GM) animals are to change the characteristics of animals, to acquire new opportunities for them, to eliminate some negative qualities in the desired direction for humans. Using genetic engineering methods, it's possible to obtain animals with new qualities like resistance to various diseases, increased productivity, changed characteristics of milk, meat, blood, etc. More than a dozen biologically active proteins have already been obtained from GM animals (cows, pigs, goats, rabbits, and sheep). As living self-replicating bioreactors, they are much more cost-effective than biotechnological or chemical production of similar substances. Transgenic animals that secrete with milk valuable proteins, such as antibodies, enzymes, blood clotting factors, and others, are increasingly used as bioreactors-producers of biologically active human proteins. Genetically modified animals are also used in biomedicine for studying the molecular basics of human diseases and for modeling diseases (Shepelev et al., 2018). The rabbit is the smallest animal used both in laboratory practice and in animal husbandry, from which recombinant pharmaceutical proteins can be obtained on an experimental and industrial scale. In contrast to the mouse model, the rabbit has a number of advantages. It has been usedfor studying human diseases for a long time, and it is used as a biomodel for evaluating the general toxic effect of biologically active agents and pharmaceuticals. During the last two decades, rabbits, especially GM ones, are becoming increasingly popular in the field of biomedicine (Rybakova et al., 2016; Bosze \& Houdebine, 2006; Honda \& Ogura, 2017). Rabbits quickly reach sexual maturity, have a short gestation period, and produce numerous offspring with relatively simple maintenance.

From different ways to create gene modifications in animals, such as pronuclear microinjection (MI), the using of viral constructions, the using of embryonic stem cells (ESC), the method of somatic cell nuclear transfer (SCNT), MI is the most convenient and often used. The first transgenic rabbit was obtained by the classic method of microinjection of the DNA structure in the zygote pronucleus as far back as 1985, but the efficiency of creating transgenic large lowyielding animals in this way remained extremely low for a long time. In contrast to mice, the technology of using ESC practically did not work on rabbits and the SCNT method had very low efficiency (Zakhartchenko et al., 2011) The first rabbit with a knocked-out gene (KO) was obtained by SCNT method just in 2015 (Yin et al., 2015). Meanwhile, rapidly developing new endonuclease methods of genome editing made it possible to obtain animals of different species, including rabbits, with KO-genes (Honda et al., 2015).

With the advent of genomic editing methods based on new endonuclease technologies (ZFN, TALEN, and CRISPR/Cas9), the efficiency of obtaining GM animals by microinjection increased several times, this made a relatively simple method of zygotes microinjecting quite acceptable for low-yielding animals. In 2011, using ZFN (zinc-finger nucleases), there was obtained the first GM rabbit with site-specific modification-destruction of the locus of the immunoglobulin M gene (Flisikowska et al., 2011). This technology has been applied to create rabbits with knockout of Apolipoprotein C-III and Apolipoprotein E genes (Yang et al., 2013; Ji et al., 2015), cholesterol ester transfer protein (CETP) (Zhang et al., 2017) in 2017. At about the same time, using TALEN (transcription activator-like effector nucleases) technology, there were created rabbits with the knockout of protein-recombinase Rag1, Rag2 (Recombination activating gene) (Song et al., 2013) and fumaryl-acetoacetate hydrolase genes (Li et al., 2017).

From a superovulating rabbit, 30-50 oocytes/embryos can be extracted, and up to $50 \%$ of the embryos develop after their microinjection by components of one endonuclease system or another. After their transplantation to the fallopian tubes, the recipient rabbits produce 30-50\% offspring, whose GM efficiency can reach $100 \%$.

In comparison with classical transgenesis, modern technologies make it possible to obtain animals with an extended range of modifications (knockout of one or more target genes at the same time, site-specific insertion (knockin, KI) of a specific genomic sequence) in application to fertilized eggs using the MI method with very high efficiency.

The production of biologically active proteins is an important field of rabbit application, including transgenic ones: rabbit monoclonal antibodies (mAbs) from using for just scientific purposes get an increasing therapeutic and diagnostic application. Nivolumab and atezolizumab (mAbs to the PD-1: programmed cell death receptor) are demanded in targeted cancer immunotherapy.

Rabbit gene sequences have more homology with human genes than rodent genes. Early embryogenesis and gastrulation (the process of germ layers formation) of rabbits are closer to humans, what allows using them as a reproductive model: the teratogenic effect of thalidomide, which was not shown in mice, was confirmed in rabbits. Lung diseases, its pathophysiology, and inflammatory reactions of the rabbit model are comparable to those found in humans: they are used in medical tests for allergic reactions, as a model for studying stem cell therapy, and eye diseases. Modeling of tumors in rabbits has been used for a long time: VX2 tumor transplantation (squamous cell carcinoma) is carried out in experimental oncology to model malignant neoplasms of the head and neck, kidney, brain, liver, in the study of colorectal cancer with liver metastases.

Since 2013 using CRISPR/Cas9 (clustered regularly interspaced short palindromic repeats) technology there were produced GM rabbits with knockout of one or simultaneously several genes encoding more than 30 proteins; with integrated, knock-in (KI), gene modifications; with point modifications of the gene in order to knock out or change the properties of the protein encoded by it.

The ROSA26 locus is often used to create GM animals with a predictable transgene expression profile. Transcripts of this locus that do not encode RNA are expressed in all tissues. Integration of the transgene in this locus by homologous recombination in particular, does not cause adverse effects on viability and allows obtaining stable expression of the transgene throughout the body. In this way, hundreds of TG animals were created, primarily mice, and 
cell lines expressing various transgenes. For example, the expression of the green protein was carried out in all tissues of transgenic rabbits obtained as a result of site-specific integration of the enhanced green fluorescent protein (EGFP) gene into the Rosa26 locus by homologous recombination repair mechanism.

In recent years, using CRISPR/Cas9 technology there were produced GM rabbits-models of such human diseases as: cardiovascular (hypertrophic cardiomyopathy, atherosclerosis, arterial thrombosis); immune diseases, including immunodeficiency; infectious diseases; metabolic syndromes due to both nutrition and heredity; Xchromosome-related diseases - hypophosphatemia (a common cause of inherited rickets) and Duchenne Muscular Dystrophy; progeria syndrome; hermaphroditism; ectodermal dysplasia; cleft palate; Wilson's disease (copper metabolism disorder, leading to severe hereditary diseases of the Central nervous system and internal organs). Models were created to study the metabolism of lipids and lipoproteins (Koloskova et al., 2019).

The promoter regions of genes of tissue-specific proteins of cow, goat or sheep milk (casein, whey proteins) are used for producing transgenic animals-producers of BA proteins with milk as part of genetic constructs. Those transgenic animals are able to synthesize human protein exclusively in the mammary gland and secrete it into milk from which it can be extracted. Promoter sequences of the whey acidic protein (WAP) gene in mouse and rabbit milk were used as part of recombinant DNA in the production of the first transgenic animals. The protein content in rabbit milk is significantly higher than in cow's milk (14\% vs. 5\%), and up to $160 \mathrm{~g}$ of milk can be obtained from lactating rabbit per day (Maertens et al., 2006). GM rabbits are used for the production of pharmacological proteins with milk, which are not required in large quantities. In 2014, the US food and drug administration (FDA) approved Ruconest (manufactured by the Dutch pharmaceutical company Pharming Group NV), the first drug to be a recombinant human C1 esterase inhibitor designed to treat acute angioedema in patients with a hereditary disease. Recombinant human $\mathrm{C} 1$ esterase inhibitor, which is part of the drug, is extracted from the milk of transgenic rabbits obtained by classical transgenesis using the bovine casein alpha-S1 gene promoter in the gene design. The target protein content in rabbit milk reached 12 $\mathrm{g} / \mathrm{l}$ (van Veen et al., 2012).

Rotavirus is the leading cause of viral gastroenteritis in newborns, so the component proteins (VP2 and VP6) of virion can be considered as potential candidates for vaccine creation. TG rabbits were obtained, whose milk could be used as a source of rotavirus antigens, but the content of simultaneously secreted recombinant proteins VP2 and VP6 in milk was less than $1 \mathrm{mg} / \mathrm{ml}$. The viral proteins expressed were immunogenic. Rectal introduction of partially purified milk proteins VP2 and VP6NG (non-glycosylated form) with an adjuvant almost completely prevented diarrhea in mice infected with a mouse strain of the virus (Soler et al., 2005; 2007). In 2019, GM rabbits with the VP6 gene integrated into the $\beta$-casein locus (CSN2) were obtained using the CRISPR / Cas9 technology to create a rotavirus vaccine suitable for both mammary-gland-based production and milk-based introduction (Li et al., 2019). For this purpose, there were used microinjection containing Cas9 in the form of mRNA or protein, sgRNA, and a donor DNA vector with the VP6 gene into the cytoplasm of rabbit zygotes. A transgenic rabbit with the correct integration of the VP6 gene was obtained using the Cas9 protein and was used to produce an experimental milk-based rotavirus vaccine.

Whey acidic protein (WAP) is the main protein of milk whey, its content in rabbit milk is about $15 \mathrm{~g} / \mathrm{l}$ (Maertens et al., 2006), which makes the WAP gene a promising candidate for replacing the biologically active protein gene using the CRISPR/Cas9 system. The regulatory sequences of the WAP gene of mice and rabbits were used in the first classical works on transgenic animal production. Using the 5 ' region of the WAP rabbit gene in the genetic structure showed good results in classical transgenesis. The recombinant protein content in the milk of transgenic mice reached $10-20 \mathrm{mg} / \mathrm{ml}$. Integration by homologous recombination of a transgene with homologous arms to the WAP gene of a mouse or rabbit using CRISPR/Cas9 technology could ensure its operation under the control of full-size endogenous regulatory sequences of the WAP gene with the possibility of obtaining mouse models and rabbits producers of recombinant proteins with its high content in milk (Koloskova et al., 2018; Ezerskiy and Koloskova, 2019). However, there are no publications on obtaining mice and rabbits with targeted modification of the WAP gene.

The purpose of our present work was to create a plasmid containing homology arms to the sequences of the WAP rabbit gene, so that the DNA sequence of the biologically active protein could be integrated into it. If a reporter fluorescent protein gene is used as an insertion into the resulting plasmid matrix, this will allow us to evaluate visually the effectiveness of site-specific transgene integration using the components of CRISPR/Cas9 technology, followed by confirmation of the homologous recombination fact by polymerase chain reaction (PCR).

\section{Materials and methods}

All the work was performed in All-Russian Research Institute of Physiology, Biochemistry and Animal Nutrition (Borovsk, Russian Federation) in the framework of the state order 2019 on the theme 0445-2019-0030. The sequence of the WAP rabbit gene was taken from the GenBank database (entry NC_013678). The genomic DNA of the Californian rabbit breed rabbit was extracted from the tip of the ear. The selection of primers, construction of recombinant DNA, and restriction analysis were performed using the Vector NTI program. All primers, oligonucleotides, and plasmid DNA sequences were ordered from "Syntol" company <http://www.syntol.ru>. For obtaining PCR amplifications of 5'-and 3' -homology arms (5'HA and 3'HA) with rabbit genomic DNA, we used pairs of primers W5-1 (AGATCTCTGCGAGGAGCTCTGTGCCT) / W5HA-2 (TACGGCCGGATGAGACAGCGCATGG) \&W3HA-1(TTCGGCCGTGTTGTCCACCAGCTGTG)/W3-2 
(GGATCCAGATCTTTCTTCTGCGAGGCCTCTGTGCACC) with the entered sequences (underlined) for BgIII, EagI and BamHI rectrictases (fig 1).

The PCR procedure was performed on a DNA amplifier "Tertsik" ("DNA Technology LLC", Moscow). For intermediate cloning of PCR products, the pTZ57R/T plasmid and reagents of the InsTAclone PCR Cloning Kit (Thermo Scientific) were used. The transformation of competent E.coli Dh5 $\alpha$ and TG1 cells was performed using the method and reagents of the Transform-Aid Bacterial Transformation Kit with our modifications. The extraction of plasmid DNA was performed using the GeneJET Plasmid Miniprep Kit with some modifications, by the classic method of alkaline lysis, and phenol-chloroform extraction method. The quality and quantity of DNA of isolated plasmids and restriction fragments were evaluated visually in UV light after electrophoresis in agarose gel (). DNA from AG was isolated using the Gene JET Gel Extraction Kit. The size of DNA fragments in AG was evaluated with DNA Ladder Mix (Fermentas). RNA guides were selected using on-line programs CHOPCHOP $<$ http://chopchop.cbu.uib.no/>, CRISPR direct <http://crispr.dbcls.jp/>, CRISPOR v.4.8. <http://crispor.tefor.net/>. To create theCRISPR/Cas9 components, a pX330-U6-Chimeric_BB-CBh-hSpCas9 plasmid (Addgene plasmid \#42230, hereinafter - pX330) was used (Cong et al., 2013).

Rabbits were kept in the vivarium of All-Russian Research Institute of Physiology, Biochemistry and Animal Nutrition. We used female rabbits of California breed at the age of 1.5-2.5 years, weighing 2.5-3.5 kg. In order to obtain the maximum number of zygotes in donor rabbits, superovulation was induced according to the previously used scheme (Ezerskiy et al., 2013). Hormone-treated female-donors were inseminated with a California male and the zygotes were surgically removed the next day. The collected zygotes were transferred to freshly prepared drops of the manipulating medium under mineral oil after washing in drops of the same medium. Microinjection into the male pronucleus of the zygote was performed in a Fonbrune camera on a device that included an inverted microscope with Nomarsky optics (Nikon) and a set of manipulators and microinjectors (Narishiga).

For long-term zygotes cultivationin vitro to the blastocyst stage, Ham's F-10 medium with the addition of $10 \%$ fetal bovine serum ("Eurobio", France) was used. Cultivation was carried out in $40 \mathrm{~mm}$ plastic Petri dishes in drops of medium with a volume of $40 \mu 1$ under light mineral oil ("Sigma" embryo tested) in the gas phase, $5 \% \mathrm{CO} 2$ in the air, at $\mathrm{t}=37^{\circ} \mathrm{C}$. Blastocysts developed from zygotes microinjected with a mixture containing GC were visually evaluated under a fluorescent microscope under blue light. Blastocysts and embryos that stopped at late stages of development were collected one at a time in $10 \mu \mathrm{l}$ of lysing solution in microtubes and stored at $-20^{\circ} \mathrm{C}$ for following detection of transgene integration in their genome by PCR method.

\section{Results and Discussion}

Creating a pWAPcmvEGFP plasmid. Obtaining plasmids encoding Cas 9 and sgRNAs to the sites of the WAP gene

PCR amplificates of 5' - and 3' -arms of homology (5'HA and 3'HA) obtained with use of primers containing the corresponding restriction sites were cloned into the pTZ57R/T vector plasmid. The sequence of the cloned 5' region of the WAP rabbit gene showed a $100 \%$ match of the sequence with the published one. As a result, there was obtaineda pTZHArbWAP plasmid containing 5'HA and 3'HA homology arms to the WAP gene, at the junction of which the site for EagI restrictase was integrated (fig. 1).

A fragment of cmvEGFP-bGHpolyA with EagI sticky ends containing a green fluorescent protein gene under a cytomegalovirus promoter ( $\mathrm{cmv}$ ) and terminated by a polyA-sequence of the bull growth hormone gene, cut and purified from a previously created by us plasmid (Ezerskiy et al., 2009), was cloned into a prepared pTZHArbWAP. A pWAPcmvEGFP plasmid was obtained, which in circle or linearized form can be used as a reporter DNA matrix for homologous recombination with the WAP gene using CRISPR/Sas9 components. Restriction analysis of the plasmid showed that the cmvEGFP-bGHpolyA fragment integrated in it is inversely oriented. Because the cmvEGFPbGHpolyA insertion is an independent expression cassette, its orientation in this case is relevant just for subsequent PCR detection. For knockout, local indels, or major deletion of the WAP gene, guide RNAs (gRNAs) sequences were selected. The pX330-511, pX330-51-, pX330-33 and pX330-31 plasmids encoding Cas9 and gRNAs to the corresponding sites of the WAP gene were created with oligonucleotides for obtaining gRNAs processed (Ezerskiy and Koloskova, 2019) according to the protocol (Menzorov et al., 2016) (fig. 2).

\section{Microinjection of embryos: development and survival}

The effect of microinjection into zygote pronuclei of solutions with different concentrations of plasmids created by us with/without a linearized fragment of WAPcmvEGFP (1-WAPcmvEGF), separately - 1-WAPcmvEGF, on the development of rabbit embryos during in vitro cultivation to the blastocyst stage was verified. Blastocysts developed from zygotes microinjected with a mixture containing transgene (groups 3 and 4, table 1) were visually evaluated under a fluorescent microscope under blue light (fig. 3).

The survival rate of embryos in the control and experimental groups was assessed. Embryos of experimental groups that developed to the blastocyst-morula stage were selected and frozen for following analysis of possible gene modifications by PCR followed by electrophoresis in agarose and polyacrylamide gels. The results of cultivation are shown in the table (table 1 ).

In groups 1 and 2 containing plasmid form (pX330-N) of components of CRISPR/Cas9 selected to target gene WAP, no significant inhibition of embryo development was found at both selected concentrations of total plasmid 
DNA. In our case, it was 10 and $20 \mathrm{ng} / \mu \mathrm{l}$ of the solution for MI. At $20 \mathrm{ng} / \mu \mathrm{l}$, the proportion of embryos that stopped at the 2-16 cell stage increased correspondingly, and the proportion of those that developed to the morula-blastocyst stage decreased. As a rulewhen using CRISPR/Sas9 in plasmid form, researchers adhere to a concentration of about $5 \mathrm{ng} / \mu 1$. In the work of Honda et al. (Honda et al., 2015) after MI 87\% of rabbit embryos developed to pre-implantation state within a day, using $5 \mathrm{ng} / \mu \mathrm{l}$ pX330 for knockout of the tyrosinase gene. On the other hand, there is evidence of cytotoxicity of microinjection by gene-specific pX330 even in this concentration. For example, when the mouse zygote pX330 was added to the genes of the proteins Centrin 1 and Protamine 1, the proportion of those developed to the 2-cell stage was 91 and 50\%, respectively (Mashiko et al., 2013). Probably, the different toxicity of gene-specific pX330 can be explained by the level of importance of the target gene for the development of the organism: the WAP gene does not belong to the "housekeeping" genes and its function is performed only during lactation.

When adding GC to the components of CRISPR/Cas9 (group 3), as with microinjection by pure GC (group 4), 80 and $96 \%$ of cultured embryos passed the 2-cell stage, after which the embryo survival during the total cultivation time of 96 hours sharply decreased to 58 and 62\%, respectively. In groups without GC (Control, groups 1 and 2), the level of development to the blastocyst-morula stage was high - 96, 88, and 84\%, respectively.

In classical transgenesis, the integration of a transgene into the genome is random, and the number of integrated copies is unpredictable. It was assumed that in the case of homologous recombination using CRISPR/Cas9 technology, green fluorescent protein expression in the developing embryo in vitro would be an effective marker of successful site-specific integration of a single copy of the transgene.

In our work at the early stages of development, the number of luminous embryos was high (63 and $53 \%$ in groups 3 and 4), and the glow was intensive. Such embryos did not develop further. At the blastocyst-morula stage, the number of fluorescent embryos decreased ( 7 and 18\%, respectively), and individual cells glowed in them, which is typical for transgenic mosaicism.

Earlier, using classical transgenesis methods, Chrenek and colleagues noted that after microinjection of GC containing the EGFP gene under a strong cmv promoter, 66-74\% of rabbit embryos developed within 72 hours of cultivation to the morula stage, compared to $98 \%$ of intact rabbit embryos. The percentage of mosaicism was high (Chrenek et al., 2013). This is consistent with our results. Reduced embryo viability may be associated with super expression of green fluorescent protein under cmv-promoter control, but not with the traumatic effect of MI.

In our 3 and 4 groups, the number and quality of luminous embryos were comparable, which makes it difficult to get a clear answer about the effectiveness and quality of transgenesis. It should be noted that we used a linearized form of the DNA matrix with BgIII sticky ends, whereas it is considered that the DNA matrix in the plasmid form has less chance of random integration into the genome. On the other hand, a plasmid carrying a cystron unit is capable of EGFP self-expression prior to its lysis by endonucleases. The possibility of short-term EGFP expression by a functional linear GC also cannot be excluded.

We have developed a system of PCR and restriction analysis of possible genetic modifications of obtained rabbit embryos, which can answer a number of questions.

\section{Conclusions.}

The All-Russian Research Institute of Physiology, Biochemistry and Animal Nutrition has experience in creating classical gene constructions for tissue-specific expression of transgenes in the mammary gland and obtaining transgenic rabbits-producers of biologically active human proteins with milk by microinjection into the zygote pronucleus (Ezerskiy et al., 2013). We also obtained two lines of transgenic rabbits with non-specific EGFP expression (unpublished data). The use of CRISPR/Cas9 technology will allow to obtain transgenic rabbits-producers of biologically active proteins with higher efficiency. Currently, we have obtained site-specific to WAP rabbit gene components of the CRISPR/Cas9 system in plasmid form, and also a DNA matrix containing homology arms to this gene, into which a gene of a biologically active protein can be integrated (Ezerskiy and Koloskova, 2019). All this makes it quite a real task to create GM rabbits that produce recombinant proteins with milk instead of whey acidic protein.

New genome editing technologies make it possible to obtain animals with precisely specified genetic modifications with high efficiency. Rabbits as biomodels of human diseases, bioreactors-producers are becoming more and more popular object of biomedical research and pharmaceutical production. Despite the obvious success of foreign, especially Chinese, geneticists in creating gene-edited rabbits, such work in Russia is practically absent.

\section{Acknowledgements}

The work was carried out as part of the State Assignment No. 0445-2019-0030 "Create genetic constructs of the GFP gene under the cytomegalovirus (CMV) promoter for site-specific integration into the genome of rabbits and cattle using CRISPR/Cas9 technology. Get components of the CRISPR/Cas9 system for the WAP gene of rabbits and BLG gene of cattle", approved and supported by the Ministry of Science and Higher Education of the Russian Federation. It is published with the support of the Federal State Budget Scientific Institution "Federal Scientific Center for Livestock - Russian Institute of Livestock Breeding named after academician L.K. Ernst", within the framework of the program "Research of molecular-biological and physiological-embryological aspects of bioengineering technologies for improving genetic resources and creating new breeding forms of farm animals and poultry" for the period of 20152020. State registration number of Scientific and Research, Development and Technological Work is AAAA-A18$118021590132-9$. 


\section{Authors' contributions}

EV, NB carried out a complex of molecular genetic studies and research, analyzed literature data, worked with specialized computer programs, including on-line, and participated in the writing of the manuscript. TT developed and implemented the cell-embryo research program, performed data interpretation, and assisted in the drafting of the manuscript. ZO participated in in vitro experiments, surgical operations on rabbits, and work on microinjection of embryos. KE developed a research program, conducted a search and analysis of literature, compiled a manuscript, and participated in the implementation of the molecular genetic block of work. All authors approved the final version of the manuscript.

\section{Declaration of conflicting interests}

The author(s) declared no potential conflicts of interest with respect to the research, authorship, and/or publication of this article.

\section{References}

1) Bosze Z., Houdebine L.M. Application of rabbits in biomedical research: a review. World Rabbit Science. 2006. 14:1-14. Doi: https://doi.org/10.4995/wrs.2006.712.

2) Chrenek P., Bauer M., Makarevich A.V. Quality of transgenic rabbit embryos with different EGFP gene constructs. Zygote. 2011. 19(1):85-90. Doi: 10.1017/S0967199410000109.

3) Cong L., Ran F.A., Cox D., Lin S., Barretto R., Habib N., Hsu P.D., Wu X., Jiang W., Marraffini L.A., Zhang F. Multiplex Genome Engineering Using CRISPR/Cas Systems. Science. 2013. 10.1126/science.1231143 PubMed 23287718 Doi: 10.1126/science.1231143.

4) Esersky V.A., Tevkin S.I., Shevchenko V.G. Gene-engineering construction with structural gene of human lactoferrin under control of regulatory elements of bovine -casein gene with reporter gene EGFP under cytomegalovirus promoter and its influence on viability of pre-implantation rabbit embryo. Problems of biology of productive animals. 2009. 1:92-103. eLIBRARY ID: 11933597. (In Russian).

5) Ezerskiy V.A., Shishimorova M.S., Tevkin S.I., Trubitsina T.P., Koloskova E.M., Bezborodova O.A., Yakubovskaya R.I., Maksimenko S.V., Ryabykh V.P.. Integration and tissue-specific expression of human lactoferrin gene in mammary gland of transgenic rabbits. Problems of biology of productive animals. 2013. 4:33-52. eLIBRARY ID: 20929501. (In Russian).

6) Ezerskii V.A., Koloskova E.M. Genetic construction for rabbit whey acidic protein gene replacement using the CRISPR/Cas9 method. Problems of biology of productive animals. 2019. 4:22-35. Doi: 10.25687/19966733.prodanimbiol.2019.4.23-35. (In Russian)

7) Flisikowska T., Thorey I.S., Offner S., Ros F., Lifke V., Zeitler B., Rottmann O., Vincent A., Zhang L., Jenkins S., Niersbach H., Kind A.J., Gregory P.D., Schnieke A.E., Platzer J. Efficient immunoglobulin gene disruption and targeted replacement in rabbit using zinc finger nucleases. PLoSOne. 2011. 6:e21045. Doi: 10.1371/journal.pone.0021045.

8) Honda A., Hirose M., Sankai T., Yasmin L., Yuzawa K., Honsho K., Izu H., Iguchi A., Ikawa M., Ogura A. Singlestep generation of rabbits carrying a targeted allele of the tyrosinase gene using CRISPR/Cas9. Experimental Animals. 2015. 64:31-37. Doi: 10.1538/expanim.14-0034.

9) Honda A., Ogura A. Rabbit models for biomedical research revisited via genome editing approaches. Journal of Reproduction and Development. 2017. 63(5):435-438. Doi: 10.1262/jrd.2017-053.

10) Ji D., Zhao G., Songstad A., Cui X., Weinstein E.J. Efficient creation of an APOE knockout rabbit. Transgenic Research. 2015. 24:227-235. Doi: 10.1007/s11248-014-9834-8.

11) Koloskova E.M., Ezerskii V.A., Belova N.V., Kutyin I.V., Ryabykh V.P., Trubitsina T.P., Maksimenko S.V. Genetic engineered construction for substitution of mouse WAP gene by cDNA of human lactoferrin by HDR method using CRISPR/Cas9 system. Problems of biology of productive animals. 2018. 2:19-30. Doi: 10.25687/1996-6733.prodanimbiol.2018.2.19-30. (In Russian).

12) Koloskova E.M., Karkischenko V.N., Yezersky V.A., Petrova N.V., Maksimenko S.V., Matveyenko E.L. Rabbit Biomodels of Human Diseases Developed Using New Genomic Technologies. CRISPR/ Cas9 (Review). Journal Biomedicine. 2019. 15(4):12-33. https://doi.org/10.33647/2074-5982-15-4-12-33

13) Li L., Zhang Q., Yang H., Zou Q., Lai C., Jiang F., Zhao P., Luo Z., Yang J., Chen Q., Wang Y., Newsome P.N., Frampton J., Maxwell P.H., Li W., Chen S., Wang D., Siu T.S., Tam S., Tse H.F.,Qin B., Bao X., Esteban M.A., Lai L. Fumarylacetoacetate Hydrolase Knock-out Rabbit Model for Hereditary Tyrosinemia Type 1. Journal of Biological Chemistry. 2017. 292(11):4755-4763. Doi: 10.1074/jbc. M116.764787.

14) Li H., Li Z., Xiao N., Su X., Zhao S., Zhang Y., Cui K., Liu Q., Shi D. Site-specific integration of rotavirus VP6 gene in rabbit $\beta$-casein locus by CRISPR/Cas9 system. In Vitro Cellular and Developmental Biology. Animal. 2019. 55(8):586-597. doi: 10.1007/s11626-019-00382-z. 
15) Mashiko D., Fujihara Y, Satouh Y, Miyata H., Isotani A., Ikawa M. Generation of mutant mice by pronuclear injection of circular plasmid expressing Cas9 and single guided RNA. Scientific Reports. 2013. $27(3): 3355$. Doi: 10.1038/srep03355.

16) Maertens L., Lebas F., SzendröZs. Rabbit milk: a review of quantity, quality and non-dietary affecting factors. World Rabbit Science. 2006. 14:205-230. Doi: https://doi.org/10.4995/wrs.2006.565.

17) Menzorov A.G., Lukyanchikova V.A., Korablev A.N., Serova I.A., Fishman V.S. Genome editing using CRisPR/Cas9 system: a practical guide. Russian Journal of Genetics: Applied Research. 2016. 20(6):930-944. Doi: 10.18699/VJ16.214. (In Russian).

18) Rybakova A.V., Makarova M.N., Makarov V.G. Use of rabbits in preclinical studies. International Journal of Veterinary Medicine. 2016. 4:102-106. eLIBRARY ID:27594552 (In Russian).

19) Shepelev M.V., Kalinichenko S.V., Deykin A.V., Korobko I.V. Production of Recombinant Proteins in the Milk of Transgenic Animals: Current State and Prospects. Acta Naturae. 2018. 10(3):40-47. Doi:10.32607/207582512018-10-3-40-47

20) Soler E., Le Saux A., Guinut F., Passet B., Cohen R., Merle C., Charpilienne A., Fourgeux C., Sorel V., Piriou A., Schwartz-Cornil I., Cohen J., Houdebine L.M. Production of two vaccinating recombinant rotavirus proteins in the milk of transgenic rabbits. Transgenic Research. 2005. 4:833-844. https://doi.org/10.1007/s11248-0051771-0.

21) Soler E., Parez N., Passet B., Dubuquoy C., Riffault S., Pillot M., Houdebine L.M., Schwartz-Cornil I. Recombinant rotavirus inner core proteins produced in the milk of transgenic rabbits confer a high level of protection after intrarectal delivery. Vaccine. 2007. 25:6373-6380. https://doi.org/10.1016/j.vaccine.2007.06.011.

22) Song J., Zhong J., Guo X., Chen Y., Zou Q., Huang J., Li X., Zhang Q., Jiang Z., Tang C., Yang H., Liu T., Li P., Pei D., Lai L. Generation of RAG 1- and 2-deficient rabbits by embryo microinjection of TALENs. Cell Research. 2013. 23:1059-1062. Doi: 10.1038/cr.2013.85.

23) van Veen H.A., Koiter J., Vogelezang C.J., van Wessel N., van Dam T., Velterop I., van Houdt K., Kupers L., Horbach D., Salaheddine M., Nuijens J.H., Mannesse M.L. Characterization of recombinant human C1 inhibitor secreted in milk of transgenic rabbits. Journal of Biotechnology. 2012. 162(2-3):319-326. Doi: 10.1016/j.jbiotec.2012.09.005.

24) Yang D., Zhang J., Xu J., Zhu T., Fan Y., Fan J., Chen Y.E.. Production of apolipoprotein C-III knockout rabbits using zinc finger nucleases. Journal of Visualized Experiments. 2013. (81):e50957. Doi: 10.3791/50957.

25) Yin M., Jiang W., Fang Z., Kong P., Xing F., Li Y., Chen X., Li S.. Generation of hypoxanthine phosphoribosyl transferase gene knockout rabbits by homologous recombination and gene trapping through somatic cell nuclear transfer. Scientific Reports. 2015. 5:16023.Doi: 10.1038/srep16023.

26) Zakhartchenko V., Flisikowska T., Li S., Richter T., Wieland H., Durkovic M., Rottmann O., Kessler B, Gungor T, Brem G, Kind A, Wolf E, Schnieke A. Cell-mediated transgenesis in rabbits: chimeric and nuclear transfer animals. Biology of Reproduction. 2011. 84:229-237. Doi: 10.1095/biolreprod.110.087098.

27) Zhang J., Niimi M., Yang D., Liang J., Xu J., Kimura T., Mathew A.V., Guo Y., Fan Y., Zhu T., Song J., Ackermann R., Koike Y., Schwendeman A., Lai L., Pennathur S., Garcia-Barrio M., Fan J., Chen Y.E. Deficiency of Cholesteryl Ester Transfer Protein Protects Against Atherosclerosis in Rabbit. Arteriosclerosis, Thrombosis, and Vascular Biology (ATVB). 2017. 37(6):1068-1075. Doi: 10.1161/ATVBAHA.117.309114. 